\title{
MSOX crystallography and simulations to capture redox enzyme catalysis
}

Michael Alexander Hough ${ }^{1}$, Demet Kekilli ${ }^{1}$, Sam Horrell ${ }^{1}$, Kakali Sen ${ }^{1}$, Chin Yong ${ }^{2}$, Thomas W.K. Keal ${ }^{2}$, Svetlana V Antonyuk ${ }^{3}$, Robert R. Eady ${ }^{3}$, S Samar Hasnain ${ }^{3}$, Richard W. Strange ${ }^{1}$

${ }^{1}$ School Of Biological Sciences, Colchester, United Kingdom, ${ }^{2}$ Scientific Computing Department, STFC Daresbury Laboratory, Warrington, United Kingdom, ${ }^{3}$ Institute of Integrative Biology, University of Liverpool, Liverpool, United Kingdom E-mail: mahough@essex.ac.uk

Relating individual protein crystal structures to enzyme mechanisms remains a challenging goal for structural biology. The mechanisms of radiation damage to macromolecular crystals have become increasingly well-characterised [1] and attention is paid to minimising the deleterious effects. Alternatively, X-rays may be used to drive enzymes to particular redox states or intermediates. Serial crystallography using multiple crystals has recently been reported in both SR and XFEL experiments. I will describe our approach to exploit rapid, shutterless X-ray detector technology on synchrotron MX beamlines to perform low-dose serial crystallography on a single $\mathrm{Cu}$ nitrite reductase crystal, allowing 10-50 consecutive X-ray structures at high resolution to be collected, all sampled from the same crystal volume. This serial crystallography approach captures the gradual conversion of the substrate bound at the catalytic type $2 \mathrm{Cu}$ centre, from nitrite to the product, nitric oxide, following reduction of the electron transfer type $1 \mathrm{Cu}$ centre by X-ray generated solvated electrons. Significant, well defined structural rearrangements in the active site are evident in the series as the enzyme moves through its catalytic cycle, which is a vital step in the global denitrification process. We propose that such a serial crystallography approach is widely applicable for studying any redox or electron-driven enzyme reactions in a single protein crystal. It can provide a 'catalytic reaction movie' highlighting structural changes that occur during enzyme catalysis. Anticipated developments in the automation of data analysis and modelling are likely to allow seamless and near-real-time analysis of such data on-site at synchrotron crystallographic beamlines.

We describe such serial crystallographic experiments conducted at $100 \mathrm{~K}$ [2] and at the elevated cryogenic temperatures of 180-200K, exploiting previously characterised changes in solvent viscosity and dynamics. Finally, we extend our approach to crystals at room temperature, allowing a more complete protein conformational response to active site structural changes to be observed. Finally, MSOX data of copper nitrite reductase at $240 \mathrm{~K}$, and molecular dynamics simulations, have revealed protein dynamics at the type 2 copper site that are significant for its catalytic properties and for the entry and exit of solvent or ligands to the active site. We performed molecular dynamics simulations using different protonation states for the key catalytic residues (AspCAT and HisCAT) involved in the nitrite reduction mechanism of this enzyme. Taken together, the crystal structures and simulations show that the AspCAT protonation state strongly influences active site solvent accessibility, while the dynamics of the active-site 'capping residue' (IleCAT), a determinant of ligand-binding, is influenced both by temperature and by the AspCAT protonation state. DFT calculations also show that the loss of a bound water ligand at the active site during the MSOX series is consistent with reduction of the type 2 copper atom.

1. Garman, E.F. \& Weik, M. (2015). J. Synchrotron Rad, 22, 195-200.

2. Horrell, S., Antonyuk, S. V., Eady, R. R., Hasnain, S S., Hough, M. A. \& Strange, R. W. (2016) IUCr], 3, $271-281$.

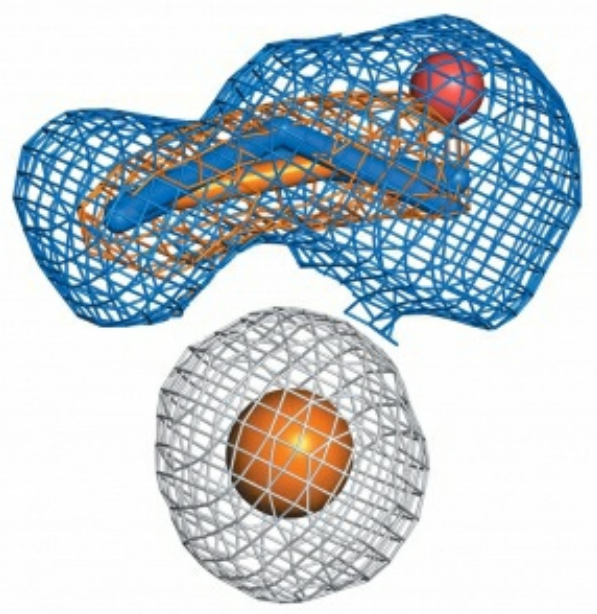

Keywords: serial crystallography, enzyme kinetics, dynamics 